\title{
VIVÊNCIA DO FAMILIAR ACOMPANHANTE DIANTE DA RESPONSABILIDADE NO CUIDADO À PESSOA IDOSA HOSPITALIZADA
}

\section{Larissa Coelho Barbosa'}

Jacilene Santiago do Nascimento Trindade dos Santos ${ }^{2}$

Rudval Souza da Silva ${ }^{2}$

Darci de Oliveira Santa Rosa² http://orcid.org/0000-0001-8978-0979 http://orcid.org/0000-0001-9535-5565 http://orcid.org/0000-0002-7991-8804

http://orcid.org/0000-0002-5651-2916

Objetivo: compreender a vivência do familiar acompanhante diante da responsabilidade no cuidado à pessoa idosa hospitalizada. Método: pesquisa descritiva com abordagem qualitativa e enfoque humanista, existencial e personalista, realizada num hospital público de Salvador - Bahia, onde foram entrevistados doze familiares acompanhantes. Resultados: apontam que os familiares passam a vivenciar outras responsabilidades, além daquelas do seu cotidiano, a exemplo de assumir novos compromissos financeiros. Todavia, o cuidado não é visto como uma obrigação, mas como gratidão, prazer, ato de amor e dedicação. Enfim, um misto de sentimento da liberdade pelo cuidar associada à responsabilidade. Conclusão: embasado na análise da Triádica-Humanista-Existencial-Personalista foi possivel compreender que os familiares personalizam o cuidado em duas vertentes humanistas, da responsabilidade e dos valores, diante da sua vivência como cuidador familiar de uma pessoa idosa hospitalizada.

Descritores: Percepção; Família; Hospital; Idoso; Poder Familiar.

\section{EXPERIENCE OF THE FAMILY COMPANION BEFORE THE RESPONSIBILITY IN THE CARE WITH THE ELDERLY INPATIENT}

Objective: to understand the experience of the family companion before the responsibility in the care with the elderly inpatient. Method: descriptive research with qualitative, humanist, existential and personalist approach, carried out in a public hospital in Salvador - Bahia, where twelve family companions were interviewed. Results: they point out that relatives begin to experience other responsibilities, besides those of their daily lives, such as assuming new financial commitments. However, care is not seen as an obligation, but as gratitude, pleasure, act of love and dedication. Finally, a mixture of feeling of freedom by caring associated with responsibility. Conclusion: based on the analysis of the Triadic-Humanist-Existential-Personalist, it was possible to understand that relatives personalize care in two humanistic aspects, of responsibility and values, given their experience as a family caregiver of an elderly inpatient. Descriptors: Perception; Family; Hospital; Aged; Parenting.

\section{EXPERIENCIA DEL FAMILIAR ACOMPAÑANTE EN VISTA DE LA RESPONSABILIDAD EN EL CUIDADO DELANCIANO HOSPITALIZADO}

Objetivo: entender la experiencia del familiar acompañante frente a la responsabilidad en la atención del anciano hospitalizado. Método: investigación descriptiva con enfoque cualitativo, humanista, existencial y personalista, realizada en un hospital público de Salvador - Bahía, donde se entrevistaron doce familiares acompañantes. Resultados: señalan que los familiares comienzan a experimentar otras responsabilidades, además de las de su vida diaria, como asumir nuevos compromisos financieros. Sin embargo, el cuidado no es visto como una obligación, sino como gratitud, placer, acto de amor y dedicación. Por último, una mezcla de sentimiento de libertad al cuidar asociada con la responsabilidad. Conclusión: sobre la base del análisis del Tríadico-Humanista-Existencial-Personalista, fue posible entender que los familiares personalizan la atención en dos aspectos humanísticos, de responsabilidad y valores, dada su experiencia como cuidador familiar del anciano hospitalizado.

Descritores: Percepción; Familia; Hospital; Anciano; Responsabilidad Parental.

IUniversidade Federal da Bahia, BA, Brasil.

${ }^{2}$ Universidade do Estado da Bahia, BA, Brasil.

Autor correspondente: Larissa Coelho Barbosa - Email: laracbarbosa@gmail.com Recebido: 02/03/2020 - Aceito: 31/07/2020 


\section{INTRODUÇÃO}

Considerar que o mundo caminha a passos largos para o envelhecimento populacional é uma realidade impactante. Segundo a Organização Mundial da Saúde (OMS), atualmente tem-se 125 milhões de idosos com mais de 60 anos de idade e, em 2050, há uma estimativa de cerca de 2 bilhões de idosos. No Brasil, esse número foi superior a 30,2 milhões no ano de 2017, com um crescimento de $18 \%$ nos últimos cinco anos². Na Bahia, para o ano de 2030, há uma perspectiva de quase 3 milhões de idosos e, com enfoque para a capital baiana, cuja população idosa já cresceu $453 \%$ nos últimos 40 anos $^{2}$.

Em contrapartida, as modificações sociais, como a crescente participação das mulheres no mercado de trabalho e a redução do número de filhos modificaram as estruturas familiares e as formas de cuidado. Em face de tal realidade, o papel de cuidador, principal, ainda é assumido pela mulher, com idade entre 18 a 80 anos, na condição, principalmente, de filha e de cônjuge, além de poder ser também de neta da pessoa idosa ${ }^{3}$. No entanto, um estudo realizado nas Unidades de Saúde de Porto Alegre/RS evidenciou que $74 \%$ dos cuidadores eram mulheres, $63 \%$ eram filhas de suas mães idosas e $54 \%$ assumiam o cuidado em tempo integral e se sentiam sobrecarregadas por serem as cuidadoras principais ${ }^{4}$.

Sobre o ato de cuidar do idoso hospitalizado, os especialistas apontam que tal ato causa estresse, esgotamento mental, físico e sobrecarga do familiar. A respeito de recursos financeiros, as pesquisas demonstraram que $64,28 \%$ dos participantes não recebem auxilio financeiro para o cuidado da pessoa idosa. Além disso, a idade média dos cuidadores é de 49,92 anos, sendo que $57,14 \%$ destes, prestam cuidados aos seus pais ${ }^{5}$

Diante desta problemática, a Portaria do Ministro da Saúde no 280/99 torna obrigatória a presença do acompanhante para pessoas com idade igual ou superior a 60 anos $^{6}$. No Brasil, os artigos 229 e 230 da Constituição Federal preconizam que os filhos maiores têm o dever de ajudar seus pais no processo de envelhecimento ou adoecimento. Quanto às políticas públicas, a Política Nacional de Saúde da Pessoa Idosa estabelece a priorização do atendimento do idoso por sua própria família?.

Portanto, entender as limitações desse familiar, cuidador, pode ajudar a melhorar a qualidade do cuidado, uma vez que um dos fatores significativos de equilibrio e bem-estar daqueles que envelhecem é a relação de afeto no ambiente familiar. Assim, destaca-se, nessa pesquisa a importância de que a presença de um idoso hospitalizado, implica para a família, mudanças na sua dinâmica, de forma a assegurar as demandas de cuidado bem como a manutenção e a integridade física e psicológica do familiar acompanhante ${ }^{8}$.

\section{OBJETIVO}

Compreender a vivência do familiar acompanhante diante da responsabilidade no cuidado à pessoa idosa hospitalizada.

\section{MÉTODO}

\section{Tipo de Estudo}

Pesquisa exploratória e descritiva, utilizando-se da abordagem qualitativa, com enfoque humanista, existencial e personalista. A abordagem qualitativa contempla todas as dimensões do "sujeito", bem como trabalha com a subjetividade, pois permite flexibilidade, não atribuindo, dessa forma, determinada padronização, o que facilita a interação com pequenos grupos ${ }^{9-10}$.

\section{Participantes da pesquisa}

Participaram da pesquisa 12 familiares acompanhantes que concordaram que atenderam aos seguintes critérios de inclusão: ser familiar importante para o idoso, independente de laços de consanguinidade; estar presente há mais de cinco dias na instituição e ser maior de 18 anos. Foram excluidos os acompanhantes contratados como cuidador formal. As entrevistas foram encerradas, quando seus relatos se tornarem repetidos, no qual se constatou o ponto de saturação nas suas falas ${ }^{11}$.

\section{Local da Pesquisa}

O cenário do estudo foi um hospital público da cidade de Salvador - Bahia, selecionado por ser referência na assistência à população menos favorecida - inclusive de outros estados. Tal hospital mantém convênio com a Secretaria de Saúde do Estado da Bahia (SESAB) e registra uma média de 16,5 mil internações e 10 mil cirurgias anuais. Atualmente com 373 leitos e um Centro de Tratamento Intensivo, o hospital oferece atendimento em 17 especialidades, divididas entre as enfermarias de Clínica Médica, Clínica Médica de Longa Permanência (crônicos) e Clínica Cirúrgica.

\section{Coleta de dados}

A coleta de dados ocorreu entre os meses de agosto a outubro de 2019, mediante entrevista aberta, individual, gravadas e transcritas, que foi orientada por roteiro semiestruturado. As entrevistas, abertas, foram guiadas pela questão norteadora: Como está sendo para você acompanhar o seu familiar idoso no hospital?

\section{Procedimento de análise dos dados}

A análise de dados seguiu o referencial teórico-metodológico da Triádica-Humanista-Existencial-Personalista, a qual se propõe a analisar as vivências como alternativa metodológica. O método preconiza o seguimento de seis etapas: 1) leitura flutuante e releitura dos relatos; 2 ) identificação das unidades de significado; 3) busca das locuções de efeito que favorecesse o reconhecimento de convergência entre as falas; 4) categorização de seus significados, 5) elaboração das subcategorias por convergência de sentido e, por fim, 6) busca da convergência de sentidos para a construção das categorias empíricas ${ }^{12}$.

\section{Aspectos éticos}

O estudo está vinculado ao projeto submetido no Comitê de Ética em Pesquisa (CEP) da instituição sob Parecer de 
no. 3.526.127 com assinatura do Termo de Consentimento Livre e Esclarecido (TCLE). Sendo assim, elegem-se pseudônimos de pedras preciosas para a identificação dos familiares.

\section{RESULTADOS}

Da análise dos dados, emergiu a Categoria Temática VIVÊNCIAS NO ACOMPANHAMENTO A IDOSOS HOSPITALIZADOS e que se apresenta em duas subcategorias: Vivenciando a responsabilidade do familiar no cuidado a pessoa idosa hospitalizada; Vivenciando valores e o sentimento da liberdade do cuidar.

\section{Categoria - VIVÊNCIAS NO ACOMPANHAMENTO A IDO- SOS HOSPITALIZADOS}

A categoria leva em consideração duas vertentes: da responsabilidade e dos valores, além de dar ênfase a vivência dos familiares diante do cuidado a pessoa idosa hospitalizada.

Subcategoria 1- Vivenciando a responsabilidade do familiar no cuidado a pessoa idosa hospitalizada

Nas unidades de significados a seguir, os familiares relatam que, mesmo com outros irmãos, vivenciam sua responsabilidade diante do cuidado da pessoa idosa hospitalizada, além de assumirem outras responsabilidades.

[...] Só mesmo a filha e esses dois netos e aí não tem contato nenhum com a família, porque assim só é nós duas, que somos responsáveis por ela (Ágata).

Porque assim no caso, ela tem muitos filhos, na hora nenhum vem. Mas assim, se veio o responsável para vender a casa, por que não veio agora que ela está internada? Entendeu? (Prata).

No que se refere à situação socioeconômica, os familiares vivenciam responsabilidades que ultrapassam os limites hospitalar. Situações de empréstimos bancários, discussões entre irmãos devido às finanças e estrutura de casa diferente do hospital trazem sofrimento aos participantes.

Vai ser muita briga, quando ela for para casa e eu não quero. [...]. Foram $25 a n o s$ tomando conta de minha mãe e de meu pai. Então, a pensão dela, eu que estou tomando conta. [...] eu tenho uma filha de três anos que fica no colégio também, fica na creche, quando eu chego pego, que precisa de mim (Prata).

Porque eu trabalho com doces e salgados e tenho curso. Então eu só venho agora quinta, eu faço encomenda, eu faço tudo que você imaginar, aí saio vendendo na rua mesmo (Ouro).

É dificil porque, ele é aposentado e eu não trabalho, eu vivo dele, dele e da filha dele. Porque ele fez uns empréstimos [...], quando soma tudo dá uns 300 e pouco, mas para ele que ganha I salário, é muito (Cornalina).
A vivência relacionada à responsabilidade do familiar no cuidado a pessoa idosa, durante a internação hospitalar, significa mais do que uma obrigação, é uma gratidão, um prazer, um ato de amor e de dedicação.

Significa tudo, me sinto bem, não faço mais porque eu não posso. Eu tenho obrigação, sou responsável por ela, porque eu não desprezo ela para nada. Eu não desprezo, aqui é uma filha minha (Jade).

Cuido dele com muito amor, significa tudo, então eu não posso deixá-lo aqui sozinho (Rubi).

Ao cuidar da pessoa idosa hospitalizada, há uma inversão de papéis familiares, no qual os filhos passam a ser responsáveis pelos pais. Nesse momento, são reveladas as vivências nas dificuldades ao assumir uma postura responsável diante do cuidado.

Porque eu assumo ainda a minha função de eletricista, ai quando há alguma necessidade, eu ai já prevendo. providencio alguém para ela não ficar só. Independente da visita, porque visita é visita, não tem nenhum compromisso, o compromisso é meu, eu sou o filho, a responsabilidade é minha. Então, quem tem que assumir sou eu, jamais deixaria ela aqui sozinha. As irmãs da igreja, fica, ora um pouquinho e tem que ir embora (Diamante).

Subcategoria 2 - Vivenciando valores e o sentimento da liberdade do cuidar

Nessa subcategoria, emergiram os valores vivenciais e o sentimento da liberdade pelo cuidar associada à responsabilidade. Os relatos, a seguir, são marcados pela crença e pela religiosidade dos familiares. Dessa forma, a fé em Deus é mantida viva, pelo familiar responsável ao cuidado da pessoa idosa hospitalizada.

Mas Ave Maria, aqui foi uma benção Meu Pai, com fé em Jesus [...]. Todo dia eu peço força à Deus para puder eu vim ver ela (Jade).

É também uma dívida que eu tenho com Deus, porque a gente tem que amar na alegria e na doença (risos). Mas como nós já somos casados há 52anos [...] Já passamos por muita coisa, altos e baixos. Mas ele sempre me respeitou, me trata muito bem. Sempre me deu tudo, sempre me dá. Então, até na hora que Deus quiser [...] (Rubi).

A gente não espera que aconteça uma coisa dessas com o nosso pai. Eu só peço a Deus, a Misericórdia, a Misericórdia Divina e força para eu aguentar (sic) tomar conta dele (Ametista).

Deus dá força, porque a gente não imagina que vai acontecer isso com a gente. Porque infelizmente, por mais força, por mais fé que nós tenhamos, nós ainda ficamos com aqueles pensamentos negativos, que existe as sequelas. Onde existe procedimentos mais grave [...] (Diamante). 
Em relação a sua liberdade, os familiares revelam que se sentem aprisionados por não poder trabalhar, estudar ou cuidar de si. Acrescentam o quanto é difícil e desgastante acompanhar o idoso no hospital, devido aos seus problemas de saúde, tais como diabetes, hipertensão e questões vasculares. Dessa forma, expressa seus sentimentos de perda da liberdade diante da impossibilidade de fazer o que deseja.

Aqui ela está quieta, em casa ela não dorme a noite, grita muito, aqui ela não grita, ela não tira a fralda, eu quero ver lá em casa. Eu vou ficar mais presa, entendeu? [...]. Já tem 1 ano que eu não vou no médico. E eu também preciso tomar conta de minha vida. E eu vou cuidar da minha vida, porque minha vida está parada. [...] Senão eu vou me deixando, o tempo passa e não volta atrás mais (Prata).

Você não vive mais a sua vida, você vive outra vida. Há dez anos atrás, a minha vida era outra. É uma luta. eu olho para a minha vida lá atrás, a minha vida era outra, eu trabalhava, estudava [...]. Eu mesma não estou podendo trabalhar (Ametista).

No momento tem sido um pouco difícil, porque eu tenho problema de saúde, diabetes, tenho problema de varizes ai fica difícil de acompanhar ele (Água Marinha).

Está sendo muito difícil, porque eu estou doente, sou viúva, tenho problema de pressão, aqui oh, tomo 12 remédios, viu, eu sou muito sofredora [...] (Jade).

\section{DISCUSSÃO}

Os familiares foram predominantemente do sexo feminino (83\%), pertencendo à faixa etária de 60 anos ou mais (75\%), o que demonstrou ser o cuidado, ainda muito delegado à mulher idosa. Em relação à escolaridade, 50\% dos familiares cursaram o ensino fundamental incompleto. Outro fato relevante é observado quanto ao grau de parentesco destes acompanhantes, nos quais os filhos são - em maioria -, os responsáveis pelo cuidado, seguidos por cônjuges e amigos ${ }^{13}$.

A predominância do sexo feminino, enquanto cuidadoras idosas e de baixa escolaridade, estão presentes nos estudos internacionais e também nacionais. Em Bogotá-Colômbia, um estudo mostrou que a maioria dos cuidadores tinha idade entre 50 e 84 anos, sendo, pois, $79 \%$ do sexo feminino, e, com relação ao nível de escolaridade, $62 \%$ tinham o ensino médio ${ }^{14}$.

Pesquisa ${ }^{15}$ com cuidadores de pacientes em cuidados paliativos, no interior de São Paulo (SP), relata que a maioria dos informantes eram do sexo feminino (80\%), porém o cuidado realizado pelos filhos (as) se destacam (36\%), assim como a participação dos cuidadores com 60 anos ou mais (32\%). A faixa etária do cuidador foi de 18 a 80 anos, com mediana de 52,5 anos e os filhos são os grandes responsáveis pelo cuidado, seguidos por cônjuges, salientando que o cuidado ainda se mantém dentro da família nuclear.

O familiar acompanhante possui um papel fundamental para garantir a qualidade de vida da pessoa idosa hospitalizada. Entretanto, o estresse da sua responsabilidade é reduzido, quando há o conhecimento dos desafios relacionado à demanda diária do cuidado. Outro problema apontado pelos participantes foi o sofrimento por não poder cuidar de sua saúde, uma vez que a maioria dos familiares são mulheres idosas, portadoras de hipertensão, diabetes e doenças cardiovasculares, associados ao número de horas que participam do cuidado ao idoso hospitalizado ${ }^{16}$.

Estudos ${ }^{17}$ relatam acerca do aumento da demanda de trabalho, que não conseguem visitar o paciente todos dos dias, pois precisam cuidar das tarefas domésticas, dos netos e de suas consultas médicas. Afirmam ainda que a alta hospitalar, do paciente, facilitaria sua vida, pois não precisariam ir ao hospital para acompanhamento.

As condições socioeconômicas e seus problemas de saúde estão evidentes nos relatos dos familiares, pois nove dos 12 participantes possuem idade maior que 60 anos, ou seja, tem-se, como panorama: idosos cuidando de pessoas idosas hospitalizadas.

O fato de alguns participantes terem que gastar seus recursos com despesas relacionadas ao cuidado também pode estar relacionado com sobrecarga do familiar hospitalizado, pois $60 \%$ dos cuidadores relataram gastar os próprios recursos financeiros para cobrir custos do cuidado prestado aos idosos. Além disso, 80\% faziam atividades domésticas, 0,9\% trabalhavam de forma remunerada e $14,7 \%$ exerciam ações de cuidadoras, atividades domésticas e trabalhavam de forma remunerada ${ }^{18}$.

Resultados de pesquisas ${ }^{15}$ relataram que no Canadá, os familiares possuem apoio governamental para o cuidado prestado ao idoso. Diferente dos cuidadores brasileiros, os quais têm renda baixa ou limitada, além de apresentar altos custos financeiros gerados pelo cuidado aos idosos. Algumas vezes, o idoso tem sua aposentadoria em torno de um salário mínimo e, dessa forma, contribui para o orçamento familiar, porém os custos com materiais de higiene e de medicamentos são maiores do que esse benefício, podendo levar ao aumento da sobrecarga do familiar.

O diagnóstico clínico e as complicações apresentadas nos pacientes são vivenciados como sentimento de culpa pelos familiares. Apesar de suas perspectivas, geralmente positivas, os familiares descrevem o sentimento de culpa, quando se ausentam para resolver os problemas de casa ou do paciente ${ }^{17}$. Entretanto, a sobrecarga do familiar pode ser aliviada se os sintomas dos pacientes, especialmente dispneia, sentimentos de depressão e ansiedade estiverem reduzidos $^{19}$. Uma pesquisa realizada na Associação Maringaense de Parkinson (AMP), Maringá, Brasil revelou que o sentimento de tristeza é vivenciado pelo familiar, e está associado ao desconhecimento da doença e do agravamento físico e neurológico do paciente ${ }^{20}$.

A religião e a fé em Deus estão sempre presentes nos relatos dos familiares. Percebe-se, nesse sentido que o valor vivencial está firmado no poder religioso na esperan- 
ça da melhora clínica do paciente. Crenças religiosas, fé e amor a Deus são, portanto, fatores relevantes para os familiares, pois serve como motivação para enfrentar os momentos difíceis ${ }^{21}$.

Estudo $^{22}$ sobre as crenças e motivos religiosos dos enfermeiros os ajudam a estar presentes ao lado do paciente e a se adaptar às situações estressantes de trabalho, apesar da fadiga física e mental e dos problemas organizacionais. Nesse contexto, os enfermeiros declararam que, frequentemente, observavam pacientes orando durante os turnos. Isso deu a eles um duplo incentivo para atender ao leito dos pacientes, apesar dos cenários de superlotação e condições de fadiga no trabalho ${ }^{23}$.

Diante desse contexto, pode-se afirmar que os profissionais da Enfermagem, em especial os enfermeiros, devem promover cuidados embasados na visão holística e humanizada, incluindo o familiar e considerando que este vai estar sempre presente nas ações de cuidado à pessoa idosa hospitalizada ${ }^{24}$.

\section{Limitações do estudo}

Este estudo apresenta como limitação o seu desenvolvimento em apenas uma instituição pública hospitalar, de natureza pública, o que não permite a generalização dos resultados, assim como se sabe que os mesmos podem ter divergências se considerar os cenários de uma instituição privada.

\section{Contribuições para a prática}

Compreender a vivência do familiar acompanhante na responsabilidade do cuidado à pessoa idosa hospitalizada torna-se essencial para o processo de formação reflexiva de futuros profissionais de enfermagem e até mesmo da- queles que já atuam no campo prático. Tal aprendizagem pode possibilitar não só o embasamento teórico-prático a respaldar a compreensão do processo de cuidado da pessoa idosa hospitalizada por seu familiar, mas também o processo emocional tanto dos pacientes quanto da equipe de saúde.

\section{CONCLUSÃO}

Essa pesquisa embasada na análise da Triádica-Humanista-Existencial-Personalista possibilitou a compreensão de que os familiares personalizam o cuidado em duas vertentes humanistas, da responsabilidade e dos valores, diante da sua vivência como cuidador familiar de uma pessoa idosa hospitalizada.

Em geral, os familiares revelaram que não conseguiram visitar o paciente todos os dias, pois necessitam cuidar das tarefas domésticas, dos netos e de suas consultas médicas e contam com a expectativa da alta hospitalar, quando não terão mais essa rotina de ir ao hospital para fazer o acompanhamento. Além disso, demonstraram vivenciar outras responsabilidades, para além das já assumidas no seu dia a dia, pois a nova rotina conduzia à necessidade de assumir novos compromissos, a exemplo dos financeiros.

Enfim, cuidar da pessoa idosa hospitalizada é um ato humanitário, descrita como forma de gratidão, de prazer, de amor e de dedicação com o próximo.

\section{Contribuição dos autores:}

a) JSNTS: concepção e/ou desenho do estudo; b) LCB: coleta, análise, interpretação dos dados; c) RSS: redação e/ ou revisão crítica do manuscrito; d) DOSR: d) aprovação da versão final a ser publicada.

\section{REFERÊNCIAS}

1. World Health Organization. Ageing and health. WHO. [Internet]. 2018 [citado 2020 mar 22]. Disponivel em: http://www.who.int/news-room/ fact-sheets/detail/ageing-and-health.

2.Instituto Brasileiro de Geografia e Estatistica. Informações Estatisticas e Geocientificas. IBGE. [Internet]. 2018 [citado 2020 mar 22]. Disponivel em: https://www.ibge.gov.br/apps/populacao/projecao/

3.Anjos KF, Boery RNSO, Pereira R, Pedreira, LC, Vilela ABA, Santos, VC, Santa Rosa DO. Association between social support and quality of life of relative caregivers of elderly dependents. Ciênc. saúde coletiva [Internet]. 2015 [citado 2020 jan 12]; 20 (5): 1321-1330. Disponivel em: https://www.scielo.br/pdf/csc/v20n5/1413-8123-csc-20-05-01321. pdf. DOI: http://dx.doi.org/10.1590/1413-81232015205.14192014.

4. Mocellin D, Aires M, Fuhrmann AC, Dal Pizzol FLF, Paskulin LMG. Filial responsibility: what are the attitudes of adult child caregivers on the institutionalization of aged parents? Rev Gaúcha Enferm. [Internet]. 2019 [citado 2020 jan 12]; 40:e20180377. Disponivel em: https://www. scielo.br/pdf/rgenf/v40/en_1983-1447-rgenf-40-e20180377.pdf. DOI: http://dx.doi.org/10.1590/1983-1447.2019.20180377.

5. Faronbi, JO, Faronbi GO, Ayamolowo, SJ, Olaogun, AA. Caring for the seniors with chronic illness: the lived experience of caregivers of older adults. Archives of Gerontology and Geriatrics [Internet]. 2019 [citado 2020 mar 10]; 82:8-14. Disponivel em: https://www.ncbi.nlm. nih.gov/pmc/articles/PMC6459393/pdf/main.pdf DOI: 10.1016 / j.archger.2019.01.013.

6. Ministério da Saúde (BR). Portaria n 280, dia 07 de abril de 1999. Dispõe sobre a presença do acompanhante de pacientes maiores de 60 (sessenta) anos de idade, quando internados. Ministério da Saúde. [Internet]. 1999 [citado 2020 mar 22]. Disponivel em: http://bvsms.saude.gov.br/bvs/saudelegis/gm/1999/prt0280_07_04_1999.html.

7. Ministério da Saúde (BR). Portaria $n \cong$ 2.528, de 19 de outubro de 2006. Aprova a Politica Nacional de Saúde da Pessoa Idosa. Ministério da Saúde. [Internet]. 2006 [citado 2020 mar 22]. Dispo- 
nivel em: http://bvsms.saude.gov.br/bvs/saudelegis/gm/2006/ prt2528_19_10_2006.html.

8. Meira EC, Reis LA, Gonçalves LHT, Rodrigues VP, Philipp RR. Women's experiences in terms of the care provided to dependent elderly: gender orientation for care. Esc. Anna Nery [Internet]. 2017 fev [citado 2020 mai 12]; 21(2). Disponivel em: https://www.scielo.br/pdf/ ean/v2ln2/en_1414-8145-ean-21-02-e20170046.pdf. DOI: http:// dx.doi.org/10.5935/1414-8145.20170046.

9. Sampieri RH, Collado CF, Lucio MPB. Métodos de Pesquisa. 5o ed. Porto Alegre: Penso; 2013.

10. Yin RK. Pesquisa qualitativa do início ao fim. Porto Alegre: Penso; 2016.

11. Polit DF, Beck CTB. Fundamentos de Pesquisa em Enfermagem: avaliação de evidências para a prática da enfermagem. 9ạ ed. Porto Alegre: Artmed; 2018.

12. Vietta EP. Configuração triádica, humanista-existencial-personalista: uma abordagem teórica-metodológica de aplicação nas pesquisas de enfermagem psiquiátrica e saúde mental Rev. Latino-am. enfermagem [Internet]. 1995 [citado 2020 mar 10]; 3(1):31-43. Disponivel em: https://www.scielo.br/pdf/rlae/v3nl/v3nla04.pdf

13. Arreguy-Sena C, Marangon AMG, Gomes AMT, Melo LD, Martins R, Fontes FSL. Representações sociais sobre esquecimento e depressão por pessoas idosas: abordagem processual. Enferm. Foco [Internet]. 2020 [citado 2020 jul 07]; 11(1): 57-62. Disponivel em: http://revista.cofen.gov.br/index.php/enfermagem/article/ view/2480/704

14. Arias-Rojas M, Carreño-Moreno S, Posada-López C. Uncertainty in illness in family caregivers of palliative care patients and associated factors. Rev. Latino-am. enfermagem [Internet]. 2019 [citado 2020 mar 10]; 27: e3200. Disponivel em: https://www.scielo.br/ pdf/rlae/v27/en_0104-1169-rlae-27-e3200.pdf DOI: http://dx.doi. org/10.1590/1518-8345.3185.3200.

15. Gayoso MV, Avila MAG, Silva TA, Alencar RA. Comfort level of caregivers of cancer patients receiving palliative care. Rev. Latino-am. enfermagem [Internet]. 2018 [citado 2020 mar 10]; 26: e3029. Disponivel em: https://www.scielo.br/pdf/rlae/v26/0104-1169-rlae-26-e3029.pdf. DOI: http://dx.doi.org/10.1590/1518-8345.2521.3029.

16. Bień-Barkowska K, Doroszkiewicz H, Bień B. Silent strain of caregiving: exploring the best predictors of distress in family carers of geriatric patients. Clinical Interventions in Aging [Internet]. 2017 [citado 2020 mar 14]; 12:263-74. Disponivel em: https://www.ncbi. nlm.nih.gov/pubmed/28203067. DOI: 10.2147 / CIA.S125664

17. Greenwooda N, Poundb C, Brearleya S, Smitha R. A qualitative study of older informal carers' experiences and perceptions of their caring role. Maturitas [Internet]. 2019 [citado 2020 fev 15]; 124: 1-7. Disponivel em: https://www.sciencedirect.com/science/article/ pii/S0378512219300878. DOI: https://doi.org/10.1016/j.maturitas. 2019.03 .006

18. Araújo MGO, Dutra MOM, Freitas CCDL, Guedes TG, Souza FS, Baptista RS. Caring for the carer: quality of life and burden of female caregivers. Rev. Bras. Enferm. [Internet]. 2019 [citado 2020 mar 12]; 72 (3): 728-736. Disponivel em: https://www.scielo.br/pdf/ reben/v72n3/0034-7167-reben-72-03-0728.pdf. DOI: http://dx.doi. org/10.1590/0034-7167-2018-0334.

19. Heliodoro EA, Santa Rosa DO. Aspectos éticos e legais da morte encefálica conhecidos por estudantes de enfermagem. Enferm. Foco [Internet]. 2019 [citado 2020 fev 25]; 10 (1): 140-5. Disponivel: http:// revista.cofen.gov.br/index.php/enfermagem/article/view/1489 DOI: https://doi.org/10.21675/2357-707X.2019.v10.n1.1489

20. Padovani C, Lopes MCL, Higahashi IH, Pelloso SM, Paiano M, Christophoro R. Being caregiver of people with Parkinson's Disease: experienced situations. Rev. Bras. Enferm. [Internet]. 2018 [citado 2020 jan 12]: 71 (Suppl 6): 2628-34. Disponivel em: https://www.scielo. br/pdf/reben/v7ls6/0034-7167-reben-71-s6-2628.pdf.DOI:http://dx.doi.org/10.1590/0034-7167-2017-0008.

21. Phenwan T, Peerawong T, Tulathamkij K. The Meaning of spirituality and spiritual well-being among thai breast cancer patients: a qualitative study. Indian J Palliat Care [Internet]. 2019 [citado 2020 mar 28]; 25 (1): 119-23. Disponivel em: https://www.ncbi.nlm.nih.gov/pubmed/30820113. DOI: 10.4103/IJPC.IJPC_101_18

22. Riklikienè O, Spirgienè L, Kaselienè K, Luneckaitè Z, Tomkevičiūtè BA. Translation, cultural, and clinical validation of the Lithuanian version of the spiritual need's questionnaire among hospitalized cancer patients. Medicina [Internet]. 2019 [citado 2020 jan 14], 55(11), 738. Disponivel: https://www.mdpi.com/1010-660X/55/11/738\#cite. DOI: https://doi.org/10.3390/medicina55110738

23. Mojarad FA, Sanagoo A, Jouybari L. Exploring the experiences of oncology nurses about the factors facilitating their presence at the bedside of patients with cancer: a qualitative study. Indian $\mathrm{J}$ Palliat Care [Internet] 2019 [citado $2020 \mathrm{fev} \mathrm{19];} \mathrm{25:236-41.} \mathrm{Disponivel:} \mathrm{ht-}$ tps://www.ncbi.nlm.nih.gov/pubmed/31114110. DOI:10.4103/IJPC. IJPC_187_18

24. Frota MA, Wermelinger MCMW, Vieira LJES, Ximenes Neto FRG, Queiroz RSM, Amorim RF. Mapping nursing training in Brazil: challenges for actions in complex and globalized scenarios. Ciênc. saúde coletiva [Internet]. 2020 [citado 2020 Jan 17] ; 25(1): 25-35. Disponivel: https://www.scielo.br/pdf/csc/v25nl/en_1413-8123-csc-25-01-0025. pdf DOI: http://dx.doi.org/10.1590/1413-81232020251.27672019. 